Relations industrielles

Industrial Relations

\title{
Le travail enchaîné, par Claude Durand, Paris, Le Seuil, 1978,
} 190 pp.

\section{Gilles Dussault}

Volume 33, numéro 2, 1978

URI : https://id.erudit.org/iderudit/028877ar

DOI : https://doi.org/10.7202/028877ar

Aller au sommaire du numéro

Éditeur(s)

Département des relations industrielles de l'Université Laval

ISSN

0034-379X (imprimé)

1703-8138 (numérique)

Découvrir la revue

Citer ce compte rendu

Dussault, G. (1978). Compte rendu de [Le travail enchaîné, par Claude Durand, Paris, Le Seuil, 1978, 190 pp.] Relations industrielles / Industrial Relations, 33(2), 370-371. https://doi.org/10.7202/028877ar

Tous droits réservés @ C Département des relations industrielles de l'Université Laval, 1978
Ce document est protégé par la loi sur le droit d'auteur. L'utilisation des services d'Érudit (y compris la reproduction) est assujettie à sa politique d'utilisation que vous pouvez consulter en ligne.

https://apropos.erudit.org/fr/usagers/politique-dutilisation/ 
Cependant, cette section doit être soumise aux mêmes critiques que celles qui ont déjà été formulées. En effet, pourquoi présenter avec autant de cynisme la nature des opérations de la FCAI puisque celle-ci n'existe à peu près plus depuis 1974. L'auteur utilise donc le même procédé qu'il a déjà utilisé avec le syndicalisme international et le syndicalisme catholique. Il refuse d'avancer que la disparition de la FCAI est peut-être due, entre autres choses, à l'efficacité des amendements apportés au Code du travail en 1969. Son interprétation des raisons de la disparition de la FCAI, bien dissimulée d'ailleurs dans une note infrapaginale, en dit long sur le caractère scientifique de son ouvrage. Je cite: «De plus en plus dénoncée, la FCAI était devenue un embarras, sinon un fardeau, tant pour le gouvernement que pour le Capital. De toute façon, il existait d'autres méthodes pour affaiblir le militantisme des syndicats affiliés à la CSN et à la FTQ». (p. 250). Et, parmi ces méthodes, Levant cite la création de la CSD, la commission Cliche ainsi que «les privilèges promis par un éventuel gouvernement du Parti québécois aux bureaucrates prévoyants». (p. 250)

Le livre de Victor Levant a raté une excellente occasion de nous présenter une analyse sérieuse sur un sujet peu documenté en relations du travail: les syndicats de boutique. Au mieux, il s'agit d'une propagande anti-capitaliste déclamée sur les airs d'un refrain que l'on ne connaît que trop depuis quelques années: "si le gazon ne pousse pas sur votre terrain, c'est à cause de l'oppression du Capital sur le Travail». Au pire c'est un ouvrage d'une faiblesse méthodologique qui frise la supercherie.

Jean BOIVIN

Université Laval

Le travail enchaîné, par Claude Durand, Paris, Le Seuil, 1978, 190 pp.

Claude Durand poursuit dans ce petit livre bien fait, les réflexions dont il a régulièrement rendu compte, ces dernières années, dans Sociologie du travail dont il est l'un des principaux animateurs. En s'interrogeant sur l'évolution de l'organisation du travail industriel, il est amené à se demander comment la division du travail, que Durkheim considérait, il n'y a pas si longtemps, comme un processus naturel ou tout au moins comme un état de fait, a pu devenir «un problème social». Car les réactions ouvrières plus nombreuses et plus virulentes, à une organisation du travail qui à force de rationaliser les tâches en asséchent le contenu (organisation dont on a largement démontré qu'elle n'avait rien de «scientifique»), indiquent bien une crise de la division du travail.

Durand nous propose d'y voir un peu plus clair, en s'appuyant d'une part sur la littérature récente sur la question et d'autre part sur l'analyse de ses propres observations; celles-ci ont porté sur sept entreprises pratiquant un système d'organisation du travail à la chaîne, à des niveaux d'automatisation divers, dont quelques-unes ont tenté des expériences de restructuration des tâches.

Pour bien comprendre les rapports de travail dans l'entreprise, il faut d'abord connaître le rôle des services techniques (bureaux d'études et de méthodes, planification, contrôle) et celui des intervenants externes (ingénieurs-conseil) dans la détermination de l'organisation technique de la production. C'est à ce niveau que les principes tayloriens sont traduits dans la réalité (chap. 1 à IV). Durand observe chez les organisateurs l'adhésion assez générale à une idéologie techniciste qui fait bien peu de place aux préoccupations concernant les conditions de travail. Pourtant des phénomènes comme le freinage, la fuite du travail, les fort taux d'absentéisme et de roulement de personnel devraient suffire à convaincre les ingénieurs et autres concepteurs de l'organisation du travail de «l'échec social» du taylorisme (chap. V et VI).

C'est dans le sillage d'une reconnaissance des impasses auxquelles conduit de plus en plus l'organisation traditionnelle qu'il faut situer l'intérêt nouveau des entreprises pour la réorganisation du travail. L'auteur présente trois expériences d'enrichissement (chap. VII) et essaie d'identifier les principaux obstacles à la restructuration des tâches (chap. VIII). Ils sont le plus souvent organisationnels (résistance des cadres et des services), économiques 
(coûts directs et rentabilité incertaine) et syndicaux (attentisme, méfiance).

L'évolution de l'organisation du travail industriel depuis quelques décennies démontre, selon Durand, que la relation progrès technique-progrès social c'est-àdire "la croyance que le développement économique, lié à la mise en œuvre des techniques, conduisait inéxorablement au bien-être des hommes, voire au bonheur de l'humanité », (p. 168), est un mythe. Les effets économiques de l'organisation tylorienne du travail sont indéniables; mais ils sont aujourd'hui remis en cause par les réactions de ceux qui vivent les conditions de travail qu'elle génère. Cela indique, comme le suggérerait Illich (Illich, Yvan, La convivialité, Paris, Le Seuil, 1973) que ce type de division du travail a peut-être atteint son seuil maximal d'utilité marginale et que les effets pervers (Boudon, Raymond, Effets pervers et ordre social, Paris, P.U.F., 1978) qu'il produit en annulent les effets bénéfiques. Pour Durand, «la crise actuelle de l'organisation du travail n'est qu'un aspect de la crise plus générale de l'idéologie du progrès et de la société industrielle». C'est pourquoi «une véritable transformation des conditions de travail suppose de nouvelles formes de contrôle social».

Le travail enchaîné ajoute une pièce importante au dossier de la critique du taylorisme. Mais l'influence des principes tayloriens a débordé largement les milieux industriels pour pénétrer les bureaux; il serait intéressant d'étendre l'analyse aux chaînes bureaucratiques et administratives.

\section{Gilles DUSSAULT}

Université Laval

Dictionnaire canadien des relations du travail, par Gérard Dion, Québec, Les Presses de l'Université Laval, 1976, $662 \mathrm{pp}$.

La revue Relations industrielles, qui rend compte couramment des nouveaux ouvrages canadiens dans le vaste domaine qu'elle couvre, a fait, apparemment, l'impasse sur l' euvre la plus importante et la plus complète publiée ces derniers temps. Omission dûe, sans doute, à la réserve de son directeur, qui se trouve être, en même temps, l'auteur de l'appréciable Dictionnaire canadien des relations $d u$ travail.

Humilité qui s'ajoute à celle qui l'a inspiré dans sa démarche, «seul le souci bien profond d'être utile nous ayant poussé à affronter ce défi avec beaucoup de modestie».

Il se peut que des spécialistes soient tentés, à l'examen d'un nouveau dictionnaire de cette envergure - le premier en langue française et, par sa qualité, dans le domaine international - de procéder au jeu malicieux d'identification des termes manquants. Ce serait, pourtant, là le fruit d'une impulsion en l'occurrence excessive. Car de cette œuvre, imposante et durable, ce qui ressort d'abord c'est l'étendue de nos ignorances et le vide qu'elle contribue à combler.

Il est vrai que les dictionnaires sont faits pour être consultés. À la différence des encyclopédies. J'ai fini, pour ma part, à le lire, quoiqu'avec une «lecture» particulière. C'est une manière d'apprendre en une forme différente de celle habituelle. Cette «lecture» stimule la curiosité, et, de ce fait, en renforce la validité.

Son prix vaut aussi par l'étendue de la gamme des connaissances qu'il implique. La vision qu'a Gérard Dion des relations du travail a été exprimée à maintes reprises, implicitement ou explicitement, dans ses écrits antérieurs au Dictionnaire. Dans «Pouvoir et 'pouvoirs' dans les relations du travail» (1970), pour ne prendre que cet exemple, l'auteur précisait qu'on désigne sous le nom de relations du travail l'ensemble des rapports sociaux et économiques qui naissent à l'occasion de la production de biens économiques, et que ces relations, à la fois individuelles et collectives, se situent au confluent de l'économique, du politique, du social et du culturel. Dans la préface au Dictionnaire, il confirme cette approche d'un champ interdisciplinaire où, dit-il, l'économique, la sociologie, la psychologie et le droit embrassent l'univers du travail, tout comme les techniques d'organisation et de gestion du personnel, disciplines plus récentes. 